\title{
CMIP is oncogenic in human gastric cancer cells
}

\author{
JIANLIN ZHANG ${ }^{1}$, JIN HUANG $^{2}$, XINGYU WANG $^{1}$, WEIDONG CHEN $^{1}$, \\ QINQING TANG $^{1}$, MAOYONG FANG $^{1}$ and YEBEN QIAN ${ }^{3}$
}

\author{
${ }^{1}$ Department of Emergency Surgery, The First Affiliated Hospital of Anhui Medical University, Hefei, \\ Anhui 230022; ${ }^{2}$ Department of Pathology, The Second People's Hospital of Hefei, Hefei, Anhui 230011; \\ ${ }^{3}$ Department of General Surgery, The First Affiliated Hospital of Anhui Medical University, Hefei, Anhui 230022, P.R. China
}

Received November 15, 2016; Accepted July 17, 2017

DOI: $10.3892 / \mathrm{mmr} .2017 .7541$

\begin{abstract}
Gastric cancer is one of the most common cancers and the second leading cause of cancer-associated mortality worldwide. Recurrence, metastasis and resistance to drug treatment are the main barrier to survival of patients with advanced stage gastric cancer. Further study of the molecular mechanisms involved will improve the therapeutic options for gastric cancer. In a previous study, c-Maf was discovered as an oncogene transduced in the avian AS42 retrovirus, and was found to be overexpressed in multiple myeloma and angioimmunoblastic T-cell lymphoma. c-Maf inducing protein (CMIP) is involved in the c-Maf signaling pathway, which was reported to serve an important role in human minimal change nephrotic syndrome and in human reading and language related behavior. However, the relationship between CMIP and human gastric cancer has not yet been reported. In the present study, CMIP protein levels in gastric cancer tissues and cells were measured using immunohistochemistry and western blot analysis; the expression of CMIP protein was significantly higher in gastric cancer tissues compared with normal gastric tissues. Expression was positively associated with poorer clinical parameters, relapse-free survival and overall survival. Furthermore, using cell counting, Cell Counting Kit-8, colony formation, wound healing and Transwell assays, together with flow cytometry, CMIP depletion by RNA interference was observed to reduce the capacity of gastric cancer cells to proliferate and migrate in vitro. Furthermore, the upstream and downstream genes of CMIP were analyzed by luciferase reporter assay and reverse transcription quantitative polymerase chain reaction, which indicated that CMIP was a direct target of miR-101-3p. In addition, CMIP knockdown was observed to result in the downregulation of MDM2 and mitogen activated protein kinase (MAPK) expression
\end{abstract}

Correspondence to: Dr Yeben Qian, Department of General Surgery, The First Affiliated Hospital of Anhui Medical University, 218 JiXi Road, Hefei, Anhui 230022, P.R. China

E-mail: qianyeben@hotmail.com

Key words: c-Maf inducing protein, oncogene, proliferation, metastasis, gastric cancer at the mRNA level. In conclusion, CMIP demonstrated an oncogenic role in human gastric cancer cells. Furthermore, microRNA-101-3p, MDM2 and MAPK were involved in the CMIP signaling pathway in gastric cancer. CMIP could be a novel target for further investigation in the clinical therapeutic management of gastric cancer.

\section{Introduction}

Gastric cancer is one of the most common cancers worldwide and has the second highest mortality (1-3). In 2012 there were 951,600 new gastric cancer diagnoses and 723,100 deaths $(4,5)$. The mass screening for gastric cancer that has developed in recent years has resulted in the diagnosis of several patients with an early stage of gastric cancer, and these patients were subsequently treated in a timely fashion, with surgery or drug treatment (6). However, the majority of patients with gastric cancer were diagnosed at an advanced stage, and their mean survival period was $<1$ year $(1,7)$. Recurrence, distant metastasis and resistance to drug treatment are the main barrier to survival of patients with advanced stage gastric cancer. Therefore, further study of the molecular mechanisms of gastric cancer may improve the therapeutic options for gastric cancer.

c-Maf was discovered as an oncogene transduced in the avian AS42 retrovirus, which was discovered to be overexpressed in multiple myeloma and angioimmunoblastic T-cell lymphoma $(8,9)$. c-Maf inducing protein (CMIP) is an adaptor protein with two isoforms, which is involved in the c-Maf signaling pathway. The two isoforms of CMIP were observed to be expressed in the human brain $(10,11)$. One of the isoforms is a short protein that participates in several cell-signaling pathways and was reported to be associated with minimal change nephrotic syndrome (MCNS) (10-13). The second isoform of CMIP is a longer protein, for which there is limited functional information at present (10-13). In kidney-associated diseases CMIP regulates the behavior of podocytes $(14,15)$. Newbury et al (11) and Scerri et al (16) demonstrated that CMIP is associated with reading and language related traits. Audard et al (13) determined that CMIP contributes to classical Hodgkin lymphoma (13). However, the relationship between CMIP and human gastric cancer has not yet been reported.

The present study demonstrated that the expression of CMIP protein was significantly higher in gastric cancer tissues 
compared with normal gastric tissues. CMIP was positively associated with tumor size, lymph node metastasis, histological grade and clinical stage in gastric cancer. Patients exhibiting CMIP expression had poorer post-operative relapse-free survival (RFS) and post-operative overall survival (OS). Furthermore, CMIP promoted proliferation and metastasis of gastric cancer cells in vitro. Notably, the present study demonstrated that miR-101-3p suppresses the expression of CMIP, and CMIP increases the expression of MDM2 and mitogen activated protein kinase (MAPK). CMIP was demonstrated to serve an oncogenic role in human gastric cancer cells, and may be useful as a biomarker or for further investigation as a therapeutic target in the clinical management of gastric cancer.

\section{Materials and methods}

Clinical samples. A total of 100 paraffin-embedded surgical gastric cancer tissue specimens and 100 paraffin-embedded surgical paired normal gastric tissue specimens ( $\mathrm{n}=100$ patients; male, $n=54$; female, $n=46$; age, $60.03 \pm 8.77$ years; Table I) were collected at the First Affiliated Hospital of Anhui Medical University (Hefei, China) between January 2009 and December 2015. Patients who had undergone chemotherapy or radiation therapy prior to surgery were excluded, as were patients with rheumatic disease, acute infection, human immunodeficiency virus or other types of cancer. The pathological tumor stage was defined according to the sixth edition of the tumor-node-metastasis classification as defined according to the 2008 World Health Organization classification of tumors (17). Primary study endpoints were post-operative RFS and post-operative OS. RFS and OS were defined as the time from the date of surgery to the date of mortality from gastric cancer, or to the date of local recurrence or detection of distant metastasis, respectively. All tissue diagnoses were confirmed by permanent histology. The Institutional Review Boards of the First Affiliated Hospital of Anhui Medical University approved the protocol for the use of tissue samples from patients and the follow-up study. Every patient signed a written informed consent form.

Immunohistochemistry analysis. Immunohistochemistry (IHC) analyses of CMIP protein expression was performed using a Two-Step Histostaining kit (Fuzhou Maixin Biotech Co., Ltd., Fuzhou, China) with a polyclonal primary antibody against CMIP (1:100; cat. no. 12851-1-AP; Proteintech Group, Inc., Chicago, USA). The sections ( $4 \mu \mathrm{m})$ were deparaffinized in xylene and rehydrated in a graded series of ethanol solutions. For antigen retrieval, slides were heated in a microwave oven in $0.01 \mathrm{M}$ sodium citrate buffer $(\mathrm{pH} 6.0)$ for $20 \mathrm{~min}$. The slides were allowed to cool in the same buffer and were subsequently immersed in $3 \%$ hydrogen peroxide in methanol for $10 \mathrm{~min}$ to block endogenous peroxidase activity. Following 3 washes for 2 min each with phosphate-buffered saline (PBS), slides were incubated with primary antibody at $4^{\circ} \mathrm{C}$ overnight. The slides were rinsed in PBS as aforementioned, incubated at room temperature for $20 \mathrm{~min}$ with universal horseradish peroxidase (HRP)-conjugated detection reagent MaxVision ${ }^{\mathrm{TM}}$ HRP-Polymer goat anti-mouse/rabbit IHC kit (undiluted; cat. no. KIT-5030; Fuzhou Maixin Biotech Co., Ltd.) and rinsed again in PBS as aforementioned. Immunoreactive regions were visualized using 3,3'-diaminobenzidine tetrahydrochloride (Fuzhou Maixin Biotech Co., Ltd.). All IHC slides were counterstained with hematoxylin. Known positive samples were used as positive controls. An isotype matched negative control was performed using a Negative Control for Rabbit IgG at room temperature for $20 \mathrm{~min}$ (undiluted; cat. no. RAB-0102; Maixin Biotech Co., Ltd.).

The stained sections were visualized using a light Olympus microscope (Olympus Corporation, Tokyo, Japan) and were reviewed and scored for the expression of CMIP protein independently by two experienced pathologists who had no knowledge of the patients' identities and clinical status. Staining intensity and percentage of tissue staining were recorded: $\geq 10 \%$ tumor cells stained were considered positive for CMIP expression; $<10 \%$ tumor cells stained with any intensity was considered as negative expression.

Cell culture. The mixed human gastric tubular adenocarcinoma cell line MKN-28 was obtained from the American Type Culture Collection (Rockville, MD). MKN-28 was cultured in RPMI-1640 medium (Gibco; Thermo Fisher Scientific, Inc., Waltham, MA, USA) containing $10 \%$ fetal bovine serum (FBS; Gibco; Thermo Fisher Scientific, Inc.) and incubated at $37^{\circ} \mathrm{C}$ in a humidified atmosphere containing $5 \% \mathrm{CO}_{2}(18)$. MKN-28 cells have previously been reported to be cross-contaminated with MKN-74 cells (19), however, this had no impact on the outcome of the present study.

Small interfering (si)RNA and micro (mi)RNA transfection. Anti-CMIP siRNA/control siRNA and miR-101-3p/negative control (NC) mimics were purchased from Shanghai GenePharma Co., Ltd. (Shanghai, China). Transient transfections of siRNAs and miRNAs into $30-50 \%$ confluent cells were performed in 6-well plates using Lipofectamine 2000 (Invitrogen; Thermo Fisher Scientific, Inc.) according to the manufacturer's instructions. The concentration of siRNAs or miRNAs used was $75 \mathrm{pmol} /$ transfection. Cells were harvested $48 \mathrm{~h}$ post-transfection. The sequences were as follows: siCMIP\#1, 5'-CAAAGAAGCUCUCGCACAUTT-3'; siCMIP\#2, 5'-CUCACCUCGAAAUUCCUGATT-3'; siNC, 5'-UUCUCCGAACGUGUCACGUTT-3'; miR-101-3p mimic, 5'-UACAGUACUGUGAUAACUGAA-3' and NC mimics, 5'-UUCUCCGAACGUGUCACGUTT-3' (all Shanghai GenePharma Co., Ltd.).

Cell proliferation assay. MKN-28 cells were plated in 6-well plates and transfected with anti-CMIP siRNA or control siRNA. Cell proliferation was assessed by cell counting assay and Cell Counting Kit (CCK)-8 reagent. Cells were plated at a density of 10,000/well and the counting assay was performed on days 2, 3, 4 and 5. Briefly, cells were stained with $0.4 \%$ trypan blue for $30 \mathrm{sec}$ at room temperature and were counted using a blood cell counting board (XB.K.25; Shanghai Qiujing Biochemical Reagents and Instrument Co., Ltd., Shanghai, China). The CCK-8 assay was performed according to the manufacturer's instructions, and the absorbance was measured at $570 \mathrm{~nm}$.

Colony formation assay. A colony formation assay was performed as described previously $(20,21)$. Briefly, $60 \mathrm{~mm}$ 
dishes were coated with $0.5 \%$ agar (Sigma-Aldrich; Merck KGaA, Darmstadt, Germany), and a layer of treated MKN-28 cells (transfected with siCMIP\#1, siCMIP\#2 or siNC, and incubated at $37^{\circ} \mathrm{C}$ in a humidified atmosphere containing $5 \%$ $\mathrm{CO}_{2}$ for $48 \mathrm{~h} ; 1 \times 10^{3}$ cells/dish) mixed with $0.3 \%$ soft agar was added on top. The plates were incubated for up to 2 weeks, and the assays were stopped when the colonies were clearly visible by eye.

Flow cytometry analysis. Flow cytometry was performed to examine cell apoptosis. MKN-28 cells transfected with CMIP-siRNA or NC-siRNA were double-stained with Annexin V-fluorescein isothiocyanate and propidium iodide, and then analyzed by flow cytometry according to the manufacturer's instructions.

Wound healing assay. Cells $\left(5 \times 10^{5}\right)$ were cultured in 6-well plates (Sigma-Aldrich; Merck KGaA) in serum-free RPMI-1640 medium until $100 \%$ confluency was reached. The cell layers were scratched with a sterile $10 \mu \mathrm{l}$ tip (Sigma-Aldrich; Merck $\mathrm{KGaA}$ ), washed 3 times with PBS and then incubated in fresh serum-free RPMI-1640 medium for $24 \mathrm{~h}$. An inverted microscope (Olympus Corporation) was used to examine and image random fields.

Cell migration and invasion assays. Cell migration and invasion assays were performed in 24-well Matrigel-coated $8-\mu \mathrm{m}$ pore Transwell chambers (Corning Incorporated, Corning, NY, USA) according to the manufacturer's instructions. For the invasion assay, the upper Transwell chambers were coated with Matrigel (BD Biosciences, Franklin Lakes, NJ, USA). The Transwell inserts were left uncoated for the migration assay. The Transwell chambers were seeded with $2 \times 10^{4}$ cells that had previously been transfected with siRNAs for $24 \mathrm{~h}$, in media containing $0.1 \%$ FBS. The lower chamber contained media with $5 \%$ FBS. The chambers were collected 24 h later and cells in the lower chamber (i.e., migratory or invasive cells) were stained with Giemsa and imaged under an inverted microscope. The number of cells on each membrane was determined by counting the number of cells in ten high-power (x400) fields and determining the mean number of cells per field.

Target Scan analysis. To identify the potential miRNAs for CMIP-targeting, TargetScan release 7.1 was used (www. targetscan.org/vert_71/) (18).

Luciferase reporter assay. To perform the luciferase reporter assay, luciferase reporter plasmids (Promega Corporation, Madison, WI, USA) containing the CMIP wild type 3'untranslated region (UTR; CMIP-3'UTR-WT; seed region, AGUACUGU) or the CMIP mutated 3'UTR (CMIP-3'UTR-MUT; seed region, AACACUAC) were constructed.

Co-transfection of miR-101-3p mimic and luciferase reporter plasmid (CMIP-3'UTR-WT or CMIP-3'UTR-MUT) was performed in $1 \times 10^{6} \mathrm{MKN}-28$ cells in a 6 -well plate using Lipofectamine ${ }^{\circledR} 2000$, according to the manufacturer's instructions. The cells were washed with PBS twice and lysed using reporter lysis buffer (Promega Corporation). For the luciferase
Table I. Correlation of CMIP expression with clinicopathological parameters from gastric cancer patients.

\begin{tabular}{|c|c|c|c|}
\hline \multirow[b]{2}{*}{ Parameter } & \multirow[b]{2}{*}{$\mathrm{n}$} & \multicolumn{2}{|c|}{ CMIP expression } \\
\hline & & Positive, n (\%) & P-value \\
\hline \multicolumn{4}{|c|}{ Age (years) } \\
\hline$\leq 60$ & 53 & $39(73.6)$ & 0.708 \\
\hline$>60$ & 47 & $33(70.2)$ & \\
\hline \multicolumn{4}{|l|}{ Sex } \\
\hline Male & 54 & $35(64.8)$ & 0.083 \\
\hline Female & 46 & $37(80.4)$ & \\
\hline \multicolumn{4}{|c|}{ Tumor size $(\mathrm{cm})$} \\
\hline$\leq 5$ & 67 & $43(64.2)$ & 0.013 \\
\hline$>5$ & 33 & $29(87.9)$ & \\
\hline \multicolumn{4}{|c|}{ Lymph node metastasis } \\
\hline No & 37 & $20(54.1)$ & 0.002 \\
\hline Yes & 63 & $52(82.5)$ & \\
\hline \multicolumn{4}{|l|}{ Grade } \\
\hline I & 7 & $3(42.9)$ & 0.010 \\
\hline II & 62 & $41(66.1)$ & \\
\hline III & 31 & $28(90.3)$ & \\
\hline \multicolumn{4}{|l|}{ Stage } \\
\hline I-II & 54 & $34(63.0)$ & 0.029 \\
\hline III-IV & 46 & $38(82.6)$ & \\
\hline
\end{tabular}

CMIP, c-Maf inducing protein.

assay, $20 \mu \mathrm{l}$ cell extract and $100 \mu \mathrm{l}$ luciferase assay reagent were mixed at room temperature. The firefly luciferase activity of this mixture was then quantified using a Dual-Luciferase Reporter Assay System (Promega Corporation).

Reverse transcription-quantitative polymerase chain reaction (RT-qPCR). The RNA isolation and RT-qPCR assay were performed as described previously (20). Total RNA from the tissues and cells was extracted using an RNeasy Mini kit (Qiagen, Inc., Valencia, CA, USA) according to the manufacturer's instructions. Total RNA was treated with DNase I and purified using phenol-chloroform. RT-qPCR ofmiR-101-3p was performed using a mirVana qRT-PCR miRNA Detection kit (Invitrogen; Thermo Fisher Scientific, Inc.). The RT temperature protocol was as follows: $42^{\circ} \mathrm{C}$ for $60 \mathrm{~min}$ and $70^{\circ} \mathrm{C}$ for $10 \mathrm{~min}$. The qPCR thermocycling conditions were as follows: $95^{\circ} \mathrm{C}$ for $10 \mathrm{~min}$, followed by 40 cycles of $95^{\circ} \mathrm{C}$ for $5 \mathrm{sec}$ and $60^{\circ} \mathrm{C}$ for $30 \mathrm{sec}$, and then dissolution curve analysis at $95^{\circ} \mathrm{C}$ for $15 \mathrm{sec}, 60^{\circ} \mathrm{C}$ for $30 \mathrm{sec}$ and $95^{\circ} \mathrm{C}$ for 15 sec. U6 was also detected as the endogenous control. The primers used were as follows: miR-101-3p, forward 5'-ATG CAAGUCAAUAGUGUCAUG-3' and reverse 5'-GTGCAG GGTCCGAGGT-3'; U6, forward 5'-TGGAACGATACAGAG AAGATTAGCA-3' and reverse 5'-AACGCTTCACGAATT TGCGT-3'. The RT-qPCR for MDM2 and MAPK detection was performed using RT-qPCR kits [RT kit, PrimeScript ${ }^{\mathrm{TM}}$ RT reagent kit; cat. no. DRR037A; qPCR kit, SYBR PremixEx 
A
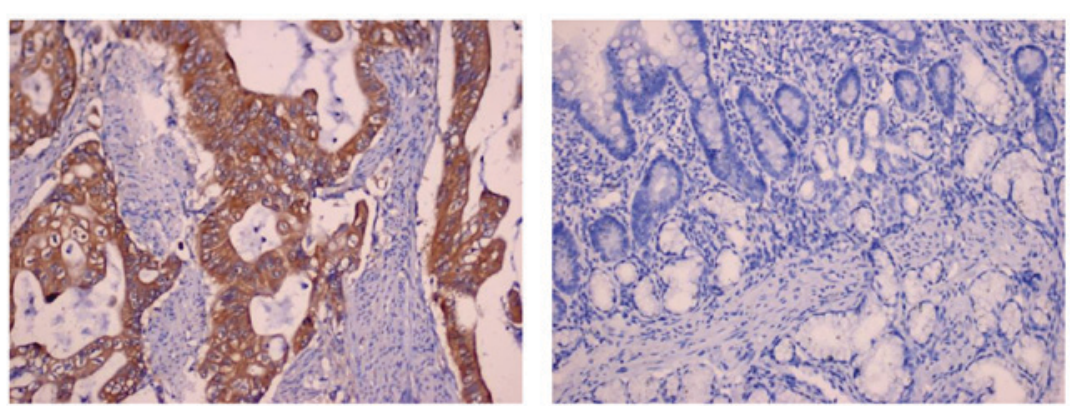

CMIP / Cancer

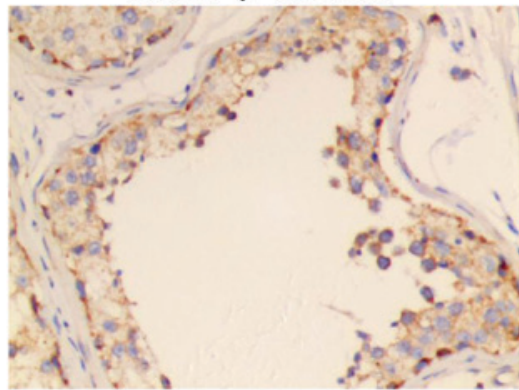

CMIP / Normal

CMIP / Positive Control

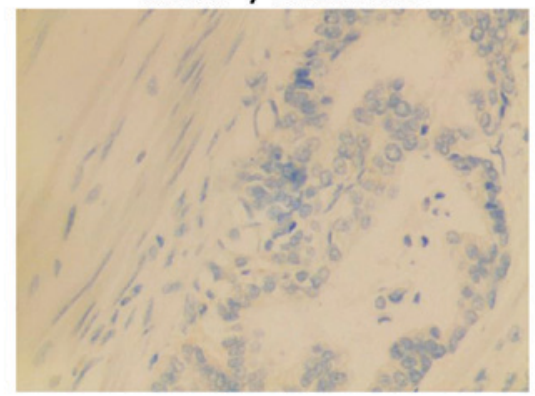

Isotype Negative Control

B
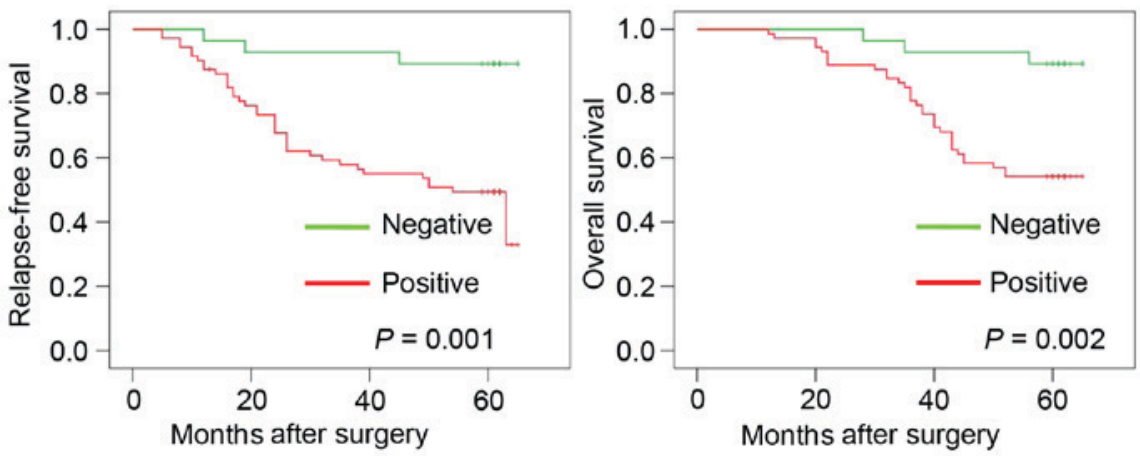

Figure 1. Expression of CMIP in gastric cancer tissues. (A) Immunohistochemistry analysis of CMIP in human gastric cancer tissues and normal gastric tissues. Human testes tissue and gastric cancer tissues were also examined as the CMIP positive and isotype negative controls, respectively. Representative images are presented (magnification, x200). (B) Association between CMIP protein expression and relapse-free survival and overall survival. CMIP, c-Maf inducing protein.

Taq ${ }^{\mathrm{TM}}$ II (Perfect Real Time); cat. no. DRR081A; both Takara Biotechnology, Co., Ltd., Dalian, China]. The RT conditions were as follows: $37^{\circ} \mathrm{C}$ for $15 \mathrm{~min}$ and $85^{\circ} \mathrm{C}$ for $5 \mathrm{sec}$. The qPCR thermo cycling conditions were as follows: $95^{\circ} \mathrm{C}$ for $10 \mathrm{~min}$, followed by 40 cycles of $95^{\circ} \mathrm{C}$ for $5 \mathrm{sec}$ and $60^{\circ} \mathrm{C}$ for $30 \mathrm{sec}$, and then dissolution curve analysis at $95^{\circ} \mathrm{C}$ for $15 \mathrm{sec}, 60^{\circ} \mathrm{C}$ for $30 \mathrm{sec}$ and $95^{\circ} \mathrm{C}$ for $15 \mathrm{sec} . \beta$-actin was also detected as the endogenous control. The primers used were as follows: MDM2, forward 5'-ACCTCACAGATTCCAGCTTCG-3' and reverse 5'-TTTCATAGTATAAGTGTCTTTTT-3'; MAPK, forward 5'-CAATGGCGGTGTGGTGTTC-3' and reverse 5'-AGCTCCCTTATGATCTGGTTCC-3'; $\beta$-actin, forward 5'-TTCCTGGGCATGGAGTC-3' and reverse 5'-CAGGTC TTTGCGGATGTC-3'. The results were quantified using the $2^{-\Delta \Delta \mathrm{Cq}}$ method (22). The mRNA levels of checkpoint kinase 2 (CHEK2), RB transcriptional corepressor 1 (RB1), B-cell lymphoma 2 (Bcl-2)-associated X (BAX), Bcl-2-associated agonist of cell death (BAD), caspase 8 and FADD-like apoptosis regulator (CFLAR), Fos proto-oncogene, AP-1 transcription factor subunit (FOS) and nuclear factor-kB1 (NF-kB1) were also determined using the same method, however, their expression levels were not associated with CMIP (data not shown). The primers used for these were as follows: CHEK2, forward 5'-AGTGGTGGGGAATAAACGCC-3' and reverse 5'-TCT GGCTTTAAGTCACGGTGTA-3'; RB1, forward 5'-GAACAT CGAATCATGGAATCCCT-3' and reverse 5'-AGAGGACAA GCAGATTCAAGGTGAT-3'; BAX, forward 5'-GGGTGG TTGGGTGAGACTC-3' and reverse 5'-AGACACGTAAGG AAAACGCATTA-3'; BAD, forward 5'-CCCAGAGTTTGA GCCGAGTG-3' and reverse 5'-CCCATCCCTTCGTCGTCC T-3'; CFLAR, forward 5'-GTGGAGACCCACCTGCTCA-3' and reverse 5'-GGACACATCAGATTTATCCAAATCC-3'; FOS, forward 5'-TGCCTCTCCTCAATGACCCTGA-3' and reverse 5'-ATAGGTCCATGTCTGGCACGGA-3'; NF-kB1, forward 5'-TGCCAACAGATGGCCCATAC-3' and reverse 5'-TGTTCTTTTCACTAGAGGCACCA-3'. 
Western blot analysis. Total protein was extracted from MKN-28 cells using the Nuclear and Cytoplasmic Protein Extraction kit (Beyotime Institute of Biotechnology, Haimen, China). Proteins $(25 \mu \mathrm{g})$ were resolved by $12 \%$ SDS-PAGE and the proteins were subsequently electrotransferred to polyvinylidene difluoride membranes (EMD Millipore, Billerica, MA, USA). Membrane blocking was performed at room temperature for $2 \mathrm{~h}$ with $5 \%$ (w/v) nonfat milk powder. Following blocking, the membranes were incubated with polyclonal primary antibodies against CMIP (cat. no. 12851-1-AP) and $\beta$-actin (cat. no. 20536-1-AP; both diluted at 1:1,000; ProteinTech Group, Inc., Chicago, IL, USA) overnight at $4^{\circ} \mathrm{C}$. The membranes were subsequently incubated with a secondary antibody [goat anti-rabbit IgG $(\mathrm{H}+\mathrm{L})$ HRP-conjugated secondary antibody; 1:50,000; cat. no. 31460; Invitrogen; Thermo Fisher Scientific, Inc.) for $2 \mathrm{~h}$ at room temperature. Protein bands were identified using an enhanced chemiluminescence system (EMD Millipore) and the substrates, Femto (cat. no. 34095; Thermo Fisher Scientific, Inc.) and Pico (cat. no. 34077; Thermo Fisher Scientific, Inc.).

Statistical analysis. All statistical analyses were performed with using SPSS for Windows (version 18.0; SPSS, Inc., Chicago, IL, USA). All data were presented as the mean \pm standard error of the mean of at least 3independent experiments. For RT-qPCR, cell counting assay, CCK-8 assay, cell colony formation assay, cell migration assay, cell invasion assay, wound healing assay, luciferase reporter assay and flow cytometry analysis, a one-way analysis of variance followed by Bonferroni or Tamhane post hoc tests were used. Pearson's chi-square test was used to analyze the results of the immunohistochemistry assay and the clinicopathological parameters. $\mathrm{P}<0.05$ was considered to indicate a statistically significant difference. Variables associated with OS and RFS rates were tested using Kaplan-Meier estimates and compared by log-rank test.

\section{Results}

Expression of CMIP in gastric cancer tissues and normal gastric tissues. The positive CMIP protein expression signals were predominantly located in the cytoplasm of the gastric cancer cells (Fig. 1A). IHC analyses were performed on 100 gastric cancer tissue specimens and 100 normal gastric tissue specimens. As presented in Table II, the positive rate of CMIP protein expression was $72(72 \%)$ in the gastric cancer tissues, whereas $48(48 \%)$ of the normal gastric tissues positively expressed CMIP $(\mathrm{P}<0.001)$.

The relationship between CMIP expression and clinicopathological parameters within gastric cancer patients. The association of CMIP expression with gastric cancer prognosis was evaluated. The clinicopathological characteristics and CMIP expression of the gastric cancer patients involved in the present study are presented in Table I. Positive expression of CMIP was associated with tumor size $(\mathrm{P}=0.013)$, lymph node metastasis $(\mathrm{P}=0.002)$, histological grade $(\mathrm{P}=0.010)$ and clinical stage $(\mathrm{P}=0.029)$ in gastric cancer.

Correlation between CMIP expression and patient survival. Kaplan-Meier estimates and log-rank tests were performed
Table II. Expression of CMIP in gastric cancer and normal tissues.

\begin{tabular}{lccc}
\hline & & \multicolumn{2}{c}{ CMIP expression } \\
\cline { 3 - 4 } Group & $\mathrm{n}$ & Negative, $\mathrm{n}(\%)$ & Positive, $\mathrm{n}(\%)$ \\
\hline Cancer & 100 & $28(28.0)$ & $72(72.0)^{\mathrm{a}}$ \\
Normal & 100 & $52(52.0)$ & $48(48.0)$ \\
\hline
\end{tabular}

${ }^{\mathrm{a}} \mathrm{P}<0.001$. CMIP, c-Maf inducing protein.

to assess whether CMIP expression was associated with post-operative RFS or OS of gastric cancer patients with complete follow-up data. The results indicated that patients with primary tumors expressing CMIP protein had a significantly poorer RFS and OS compared with those without CMIP protein expression $(\mathrm{P}=0.001$ and $\mathrm{P}=0.002$, respectively; Fig. 1B).

Effects of CMIP inhibition on cell proliferation and apoptosis of $M K N-28$ cells. To investigate the role of CMIP in MKN tumorigenesis, CMIP knockdown was performed in MKN-28 cells by transfection with CMIP-siRNA. Following transfection, CMIP-siRNA treated cells had significantly lower CMIP protein levels compared with cells transfected with control-siRNA (Fig. 2A), indicating that the CMIP knockdown was successful. The impact of CMIP knockdown on MKN-28 proliferation and growth were assessed using cell counting, CCK-8 and colony formation assays, following treatment with CMIP-siRNA (75 pmol). As presented in Fig. 2B-D, cells transfected with CMIP-siRNA exhibited a marked decrease in proliferation, viability and growth, compared with control-siRNA treated cells. Furthermore, cell apoptosis was examined using flow cytometry (Fig. 2E). Cells transfected with CMIP-siRNA had greater levels of apoptosis compared with control-siRNA treated cells (30.98\% vs. $75.98 \%)$.

Effects of CMIP inhibition on the migration and invasion of MKN-28 cells. To explore the role of CMIP in gastric cancer cell migration and invasion, the effects of CMIP knockdown on MKN-28 cells were assessed using Transwell and wound healing assays. MKN-28 cells transfected with CMIP-siRNA displayed reduced migration and invasion in Transwell and wound healing assays (Fig. 3), compared with control-siRNA treated cells.

CMIP is a direct target of miR-101-3p in MKN-28 cells. To investigate the regulation of CMIP in gastric cancer, TargetScan was used to search for potential CMIP-targeting miRNAs. The TargetScan analysis indicated that CMIP may be a target gene of miR-101-3p. The miR-101-3p-binding site was found in the wild-type 3'-UTR of CMIP, and a luciferase reporter plasmid was constructed containing the wild type binding site (CMIP-3'UTR-WT). A reporter plasmid containing a mutant 3'-UTR of CMIP (CMIP-3'UTR-MUT) was also constructed (Fig. 4A). RT-qPCR was performed to detect the miR-101-3p expression levels in gastric cancer 
A

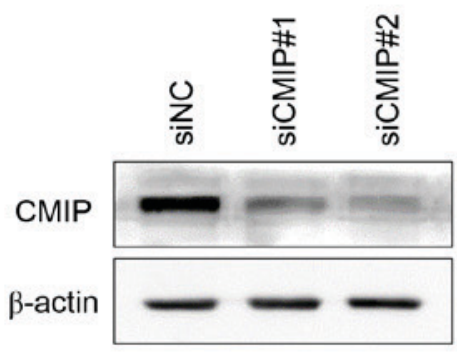

D

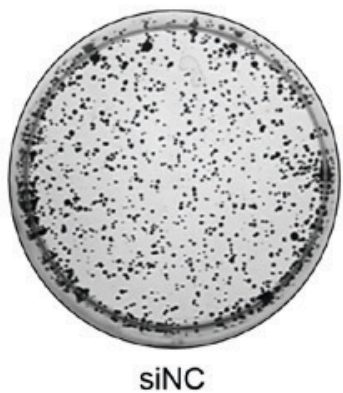

siNC
B

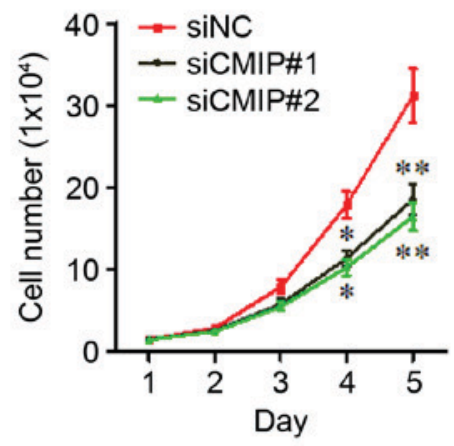

C

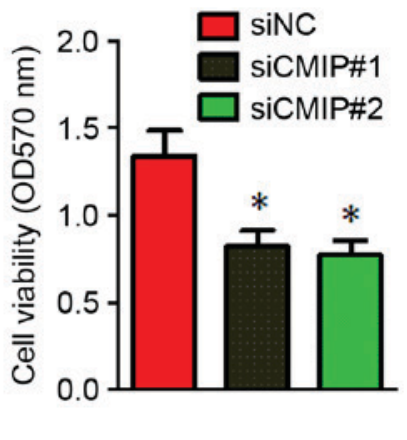

E
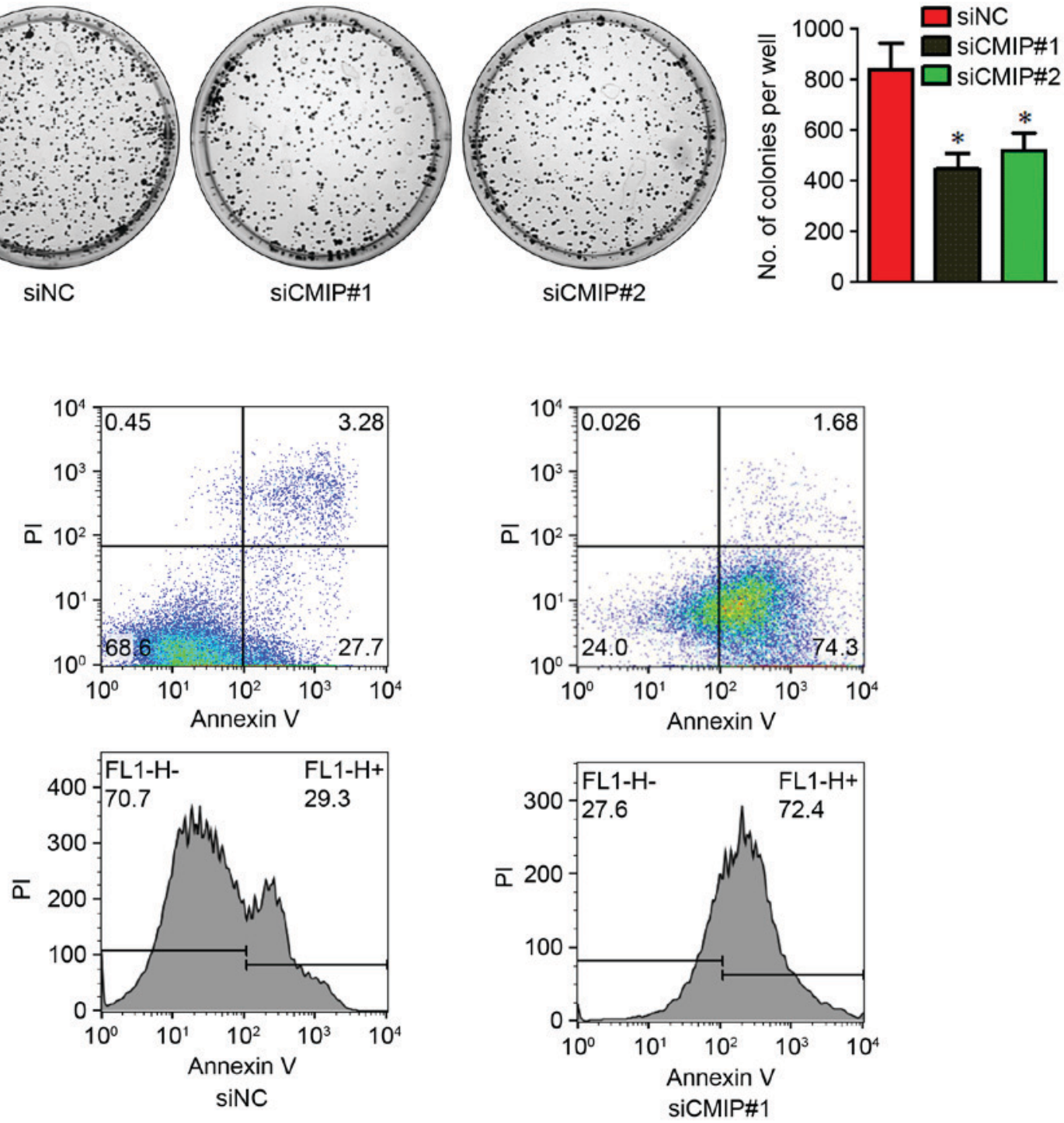

Figure 2. Knockdown of CMIP reduces the proliferation and enhances the apoptosis of MKN-28 cells. (A) The efficiency of CMIP knockdown by CMIP-siRNA was assessed by western blot analysis. Proliferation of MKN-28 cells transfected with CMIP-siRNA was measured using (B) cell counting (where Day $1=1 \times 10^{4}$ cells), (C) CCK-8 and (D) colony formation assays. (E) Apoptosis analysis of MKN-28 cells transfected with CMIP-siRNA was measured by flow cytometry using double-staining with Annexin V-fluorescein isothiocyanate and PI. CMIP, c-Maf inducing protein; si, small interfering; PI, propidium iodide; NC, negative control; OD, optical density. Data are presented as the mean \pm standard error of the mean of at least 3 independent experiments. "P<0.05 and ${ }^{* *} \mathrm{P}<0.01$ vs. siNC.

tissues and normal gastric tissues. The results indicated that miR-101-3p was significantly downregulated in gastric cancer tissues (Fig. 4B). To verify whether CMIP was a direct target of miR-101-3p, a dual luciferase reporter assay was performed. MiR-101-3p suppressed the luciferase activity of the wild type
CMIP 3'-UTR upon co-transfection of the luciferase vector in MKN-28 cells (Fig. 4C). This inhibition was reversed when the seed sequences of the miR-101-3p target sequences were mutated in the CMIP-3'UTR-MUTvector (Fig. 4C). To further investigate the effects of miR-101-3p, MKN-28 cells were 
A
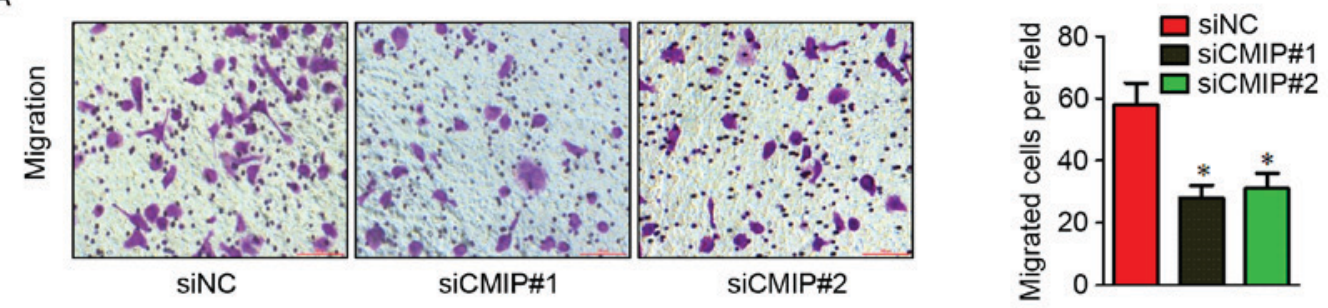

B
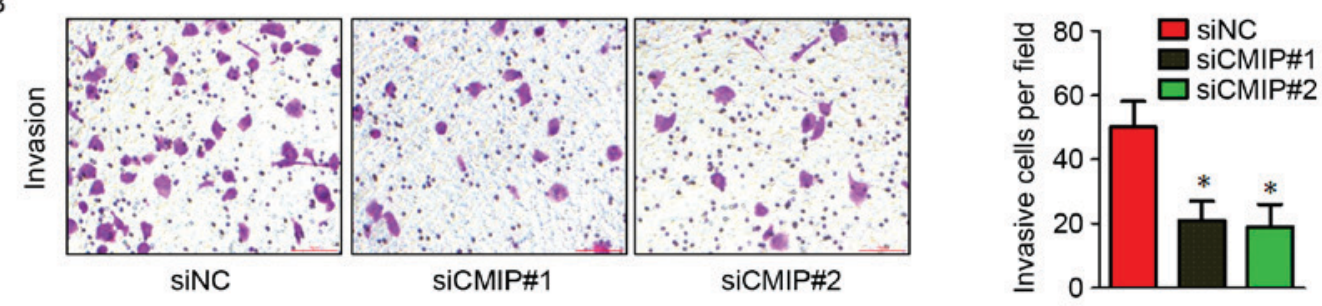

C

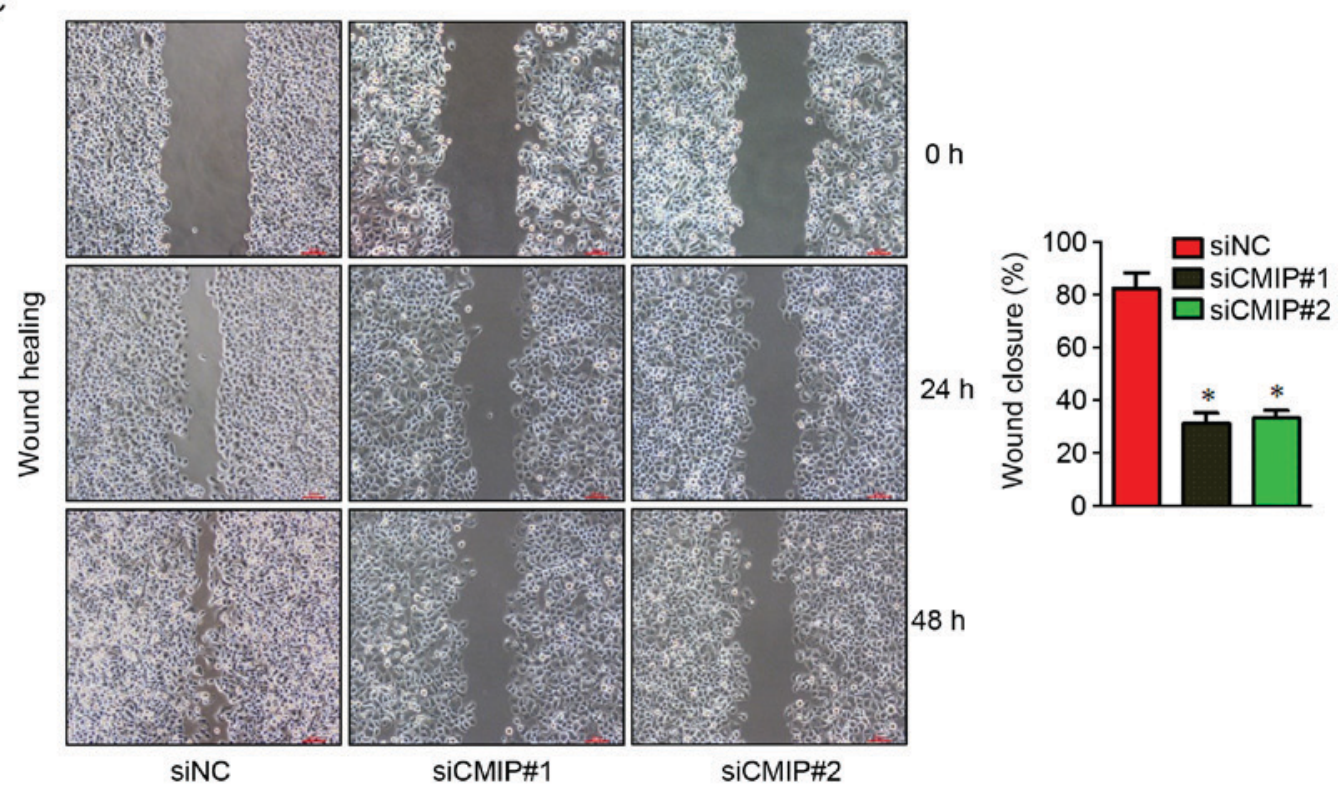

Figure 3. Knockdown of CMIP suppresses the migration and invasion of MKN-28 cells. MKN-28 cells were transfected with the indicated siRNAs and the (A) migratory and (B) invasive abilities of the cells were evaluated ( $\mathrm{n}=3$; magnification, $\mathrm{x} 100)$. (C) A scratch was made in a confluent, adherent layer of MKN-28 cells that had undergone treatment with siNC or two different siRNAs against CMIP. Cells that had migrated into the wound were counted after $24 \mathrm{~h}$ $(n=3)$. Representative images of the wounds are presented. Data are presented as the mean \pm standard error of the mean of at least 3 independent experiments. ${ }^{*} \mathrm{P}<0.05$ vs. siNC. CMIP, c-Maf inducing protein; si, small interfering; $\mathrm{NC}$, negative control.

treated with the miR-101-3p mimic. The results indicated that overexpression of miR-101-3p reduced the protein level of CMIP (Fig. 4D).

CMIP regulates MDM2 and MAPK expression in gastric cancer cells. To obtain further insight into the role of CMIP in MKN-28 cell invasion and metastasis, the expression of several mRNAs (including cell cycle control and DNA damage repair genes MDM2, CHEK2 and $\mathrm{RB} 1$, apoptosis and cell senescence genes BAX, BAD and CFLAP and signal transduction molecules and transcription factors MAPK, FOS and NFKB1) was assessed using RT-qPCR in siCMIP-transfected MKN-28 cells, compared with si-NC transfected cells (data not shown). Two downregulated genes (MDM2 and MAPK) were identified in siCMIP-transfected MKN-28 cells. The results demonstrated that mRNA levels of MDM2 and MAPK were downregulated following CMIP knockdown in gastric cancer MKN-28 cells (Fig. 4E).

\section{Discussion}

The present study documented for the first time that CMIP may serve an oncogenic role in human gastric cancer cells. In 200 samples of human gastric tissues (containing 100 cancer and 100 normal tissues), CMIP expression was significantly higher in gastric cancer tissues compared with normal gastric tissues. Patients with positive CMIP expression were associated with a larger tumor size, lymph node metastasis, higher grade, higher stage, and poorer RFS and OS. Using cell counting, CCK-8 and colony formation assays, we determined that in 
A CMIP-3'UTR-WT

miR-101-3p

CMIP-3'UTR-mut
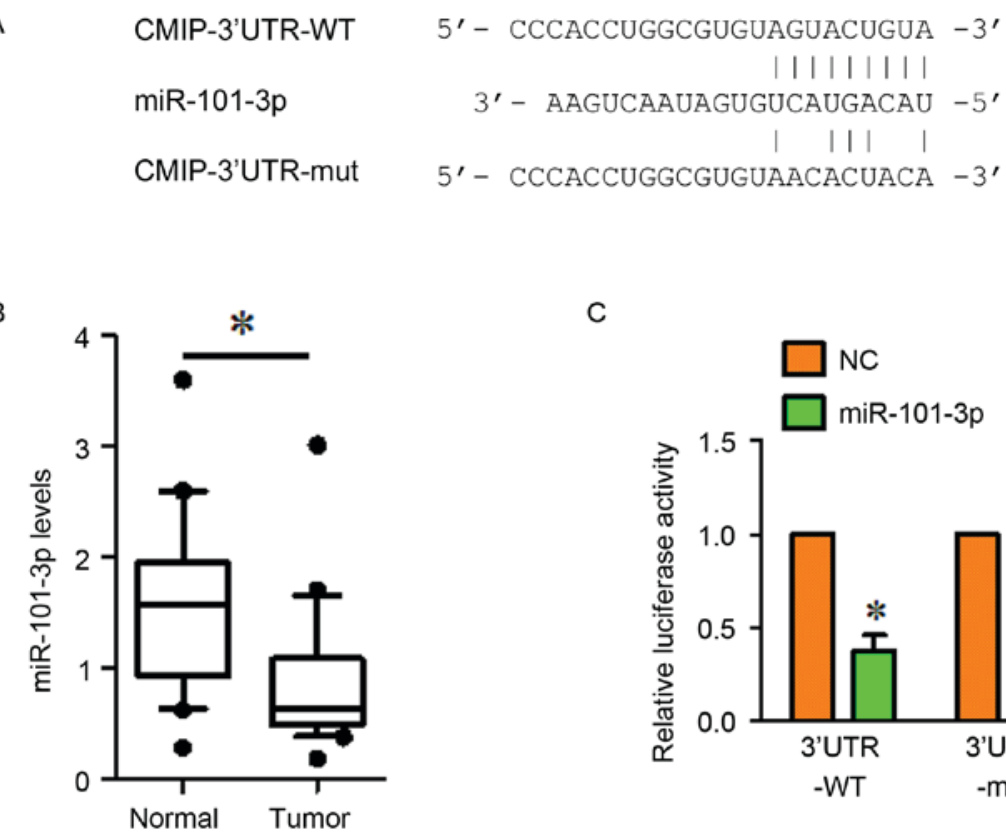

D

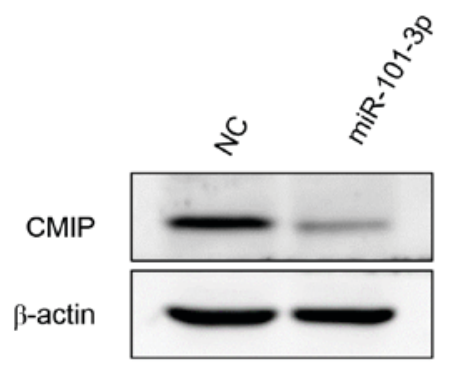

C

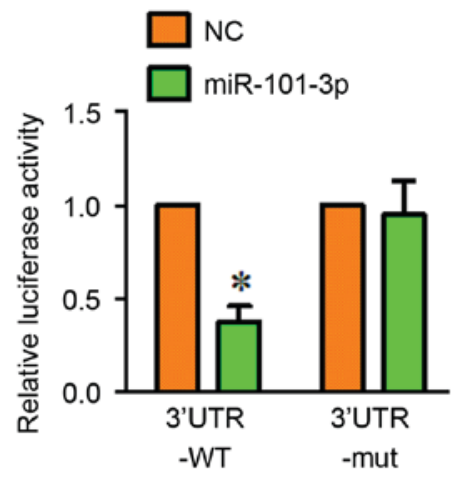

E

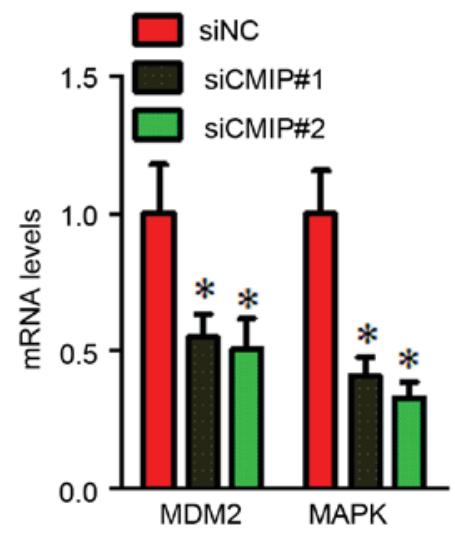

Figure 4. CMIP is a direct target of miR-101-3p and CMIP regulates the expression of MDM2 and MAPK. (A) The TargetScan-predicted binding site between miR-101-3p and the 3'-UTR of CMIP. The mutant 3'-UTR of CMIP is also presented. (B) miR-101-3p was down-regulated in gastric cancer tissues compared with normal gastric tissues. ${ }^{*} \mathrm{P}<0.05$ vs. Normal. (C) Luciferase assay of MKN-28 cells cotransfected with miR-101-3p mimic or NC, and a luciferase reporter containing CMIP 3'-UTR wildtype or mutant constructs. "P<0.05 vs. NC. (D) MKN-28 cells were transfected with miR-101-3p mimics or NC. miR-101-3p overexpression inhibited the protein expression of CMIP. $\beta$-actin served as a loading control. (E) Cells transfected with CMIP-siRNA demonstrated a significant decrease in MDM2 and MAPK mRNA expression compared with cells transfected with siNC. All data were presented as the mean \pm standard error of the mean of at least 3 independent experiments. "P<0.05 vs. siNC. CMIP, c-Maf inducing protein; MAPK, mitogen activated protein kinase; miR, microRNA; NC, negative control; si, small interfering; 3'UTR, 3-untranslated region; 3'UTR-WT, wild type 3'UTR construct; 3'UTR-MUT, mutant 3'UTR construct; MAPK, mitogen activated protein kinase.

MKN-28 gastric cancer cells, cell proliferation was markedly decreased following knockdown of CMIP. Furthermore, flow cytometry indicated that cell apoptosis was significantly increased following CMIP knockdown. Cell migratory and invasive abilities were also decreased following knockdown of CMIP in MKN-28 cells, as determined by migration, invasion and wound healing assays. The oncogene c-Maf was recently demonstrated to be overexpressed in multiple myeloma and angioimmunoblastic T-cell lymphoma $(8,9)$. Overexpression of c-Maf is a frequent oncogenic event in multiple myeloma that promotes proliferation and pathological interactions with the bone marrow stroma (23). CMIP is involved in the c-Maf signaling pathway, which serves an important role in MCNS and in human reading and language related behavior $(11-13,16)$. However, the relationship between CMIP and human carcinoma has not been well studied. Therefore, the present study examined the role of CMIP in human gastric cancer.

Upstream of CMIP, a luciferase assay indicated that CMIP may be a direct target of miR-101-3p in gastric cancer MKN-28 cells. Furthermore, CMIP was significantly reduced by overexpression with miR-101-3p. miRNAs are non-coding RNAs consisting of 20-25 nucleotides, which were identified to serve important roles in the initiation, development, growth, proliferation and metastasis of human cancer, including gastric cancer (24-26). miR-25, miR-214 and miR-132, were demonstrated to promote tumor growth or metastasis of gastric cancer (27-29), whereas miR-320a, miR-205 and miR-26b were revealed to suppress tumor proliferation, migration or invasion of gastric cancer (30-32). The present study demonstrated that 
the expression of miR-101-3p was markedly lower in gastric cancer tissues compared with normal gastric tissues, indicating that miR-101-3p may serve a tumor suppressive role in gastric cancer. miR-101-3p suppressed the expression of CMIP, and miR-101-3p and CMIP exhibited opposing expression patterns. These results supported the earlier observation that CMIP expression was elevated in gastric cancer tissues. As previously reported, miR-101-3p has demonstrated a tumor-suppressing role in human breast cancer, salivary gland adenoid cystic carcinoma and hepatocellular carcinoma (33-35), and these results are consistent with the current findings.

When investigating the downstream pathway, CMIP knockdown was revealed to downregulate the expression of MDM2 and MAPK. MDM2 has previously been identified as an oncogene in gastric cancer; MDM2 promoted tumor initiation and development by targeting and reducing tumor suppressor genes, including p53 (36,37). Furthermore, it was reported that MDM2 serves a promoting role in the migration and invasion of gastric cancer (38), and inhibition of MDM2 expression could induce the apoptosis of gastric cancer cells (39). MDM2 has also demonstrated a tumor promoting ability in breast cancer and non-small cell lung cancer $(40,41)$. MAPK was reported to serve as an oncogene in gastric cancer, where it promoted tumor growth and metastasis $(42,43)$. In breast cancer, colon cancer and hepatocellular carcinoma, MAPK contributed to tumor development, migration and invasion (44-46). These results supported the results of the present study, and suggest that CMIP may serve an oncogenic role in gastric cancer cells partly via regulating the expression of MDM2 and MAPK. Therefore, the results imply that CMIP was regulated by miR-101-3p and itself regulated MDM2 and MAPK; this signaling pathway appears to be involved in promoting a cancer phenotype in vitro and may promote the oncogenicity of gastric cancer.

The present study investigated the role of CMIP in the human gastric cancer cell line MKN-28. This is the first report on the role of CMIP in human gastric cancer. The cell line selected for the present study was MKN-28, which was previously reported to be cross-contaminated with MKN-74 cells (19). MKN-28 and MKN-74 cells have been reported to contain different genetic and epigenetic alterations of p53, p21, cluster of differentiation 44 and adenomatous polyposis coli, and different expression levels of B-cell lymphoma 2, epidermal growth factor (EGF), EGF receptor, transforming growth factor- $\alpha$, interleukin (IL)-1 $\alpha$, IL-8, vascular endothelial growth factor, cyclin $\mathrm{E}$ and platelet derived growth factor $(19,47)$. These genes have no direct relation with CMIP, the CMIP-related upstream miR-101-3p and downstream proteins MDM2 and MAPK. Therefore, use of the mixed gastric tubular adenocarcinoma cell line MKN-28 had no impact on the outcome of the study.

In conclusion, the present study demonstrated a tumor-promoting function of CMIP in the MKN-28 cell line. miR-101-3p targeted CMIP, and knockdown of CMIP downregulated MDM2 and MAPK. It is possible that this signaling pathway may contribute to the oncogenic role of CMIP in gastric cancer. In patients with gastric cancer, expression of CMIP was associated with poorer clinical parameters, RFS and OS. These results suggest that CMIP may be a novel therapeutic target for gastric cancer; however further studies are required to investigate this.

\section{Acknowledgements}

This study was supported by a grant from the Natural Science Foundation of Anhui Province (grant no. 1508085MH173).

\section{References}

1. LeeKB, Jin H, Ye S, Park BH and Kim SM: Recombinant human bone morphogenetic protein-2 inhibits gastric cancer cell proliferation by inactivating Wnt signaling pathway via c-Myc with aurora kinases. Oncotarget 7: 73473-73485, 2016.

2. Yin K, Liu M, Zhang M, Wang F, Fen M, Liu Z, Yuan Y, Gao S, Yang L, Zhang W, et al: miR-208a-3p suppresses cell apoptosis by targeting PDCD4 in gastric cancer. Oncotarget 7: 67321-67332, 2016.

3. Scott RB, Harrison J, Boulton C, Wilson J, Gregory R, Parkin S, Bain PG, Joint C, Stein J and Aziz TZ: Global attentional-executive sequelae following surgical lesions to globus pallidus interna. Brain 125: 562-574, 2002.

4. Feng T, Sun L, Qi W, Pan F, Lv J, Guo J, Zhao S, Ding A and Qiu W: Prognostic significance of Tspan9 in gastric cancer. Mol Clin Oncol 5: 231-236, 2016.

5. Torre LA, Bray F, Siegel RL, Ferlay J, Lortet-Tieulent J and Jemal A: Global cancer statistics, 2012. CA Cancer J Clin 65: 87-108, 2015.

6. Zakko L, Lutzke L and Wang KK: Screening and preventive strategies in esophagogastric cancer. Surg Oncol Clin N Am 26: 163-178, 2017.

7. Van Cutsem E, Moiseyenko VM, Tjulandin S, Majlis A, Constenla M, Boni C, Rodrigues A, Fodor M, Chao Y, Voznyi E, et al: Phase III study of docetaxel and cisplatin plus fluorouracil compared with cisplatin and fluorouracil as first-line therapy for advanced gastric cancer: A report of the V325 Study Group. J Clin Oncol 24: 4991-4997, 2006.

8. Murakami Y, Yatabe Y, Sakaguchi T, Sasaki E, Yamashita Y, Morito N, Yoh K, Fujioka Y, Matsuno F, Hata H, et al: c-Maf expression in angioimmunoblastic T-cell lymphoma. Am J Surg Pathol 31: 1695-1702, 2007

9. Benkhelifa S, Provot S, Nabais E, Eychéne A, Calothy G and Felder-Schmittbuhl MP: Phosphorylation of MafA Is essential for its transcriptional and biological properties. Mol Cell Biol 21: 4441-4452, 2001

10. Szymańska K, Szczałuba K, Lugowska A, Obersztyn E, Radkowski M, Nowakowska BA, Kuśmierska K, Tryfon J and Demkow U: The analysis of genetic aberrations in children with inherited neurometabolic and neurodevelopmental disorders. Biomed Res Int 2014: 424796, 2014.

11. Newbury DF, Winchester L, Addis L, Paracchini S, Buckingham LL, Clark A, Cohen W, Cowie H, Dworzynski K, Everitt A, et al: CMIP and ATP2C2 modulate phonological short-term memory in language impairment. Am J Hum Genet 85: 264-272, 2009.

12. Grimbert P, Valanciute A, Audard V, Pawlak A, Le Gouvelo S, Lang P, Niaudet P, Bensman A, Guellaën G and Sahali D: Truncation of C-mip (Tc-mip), a new proximal signaling protein, induces c-maf $\mathrm{Th} 2$ transcription factor and cytoskeleton reorganization. J Exp Med 198: 797-807, 2003.

13. Audard V, Zhang SY, Copie-Bergman C, Rucker-Martin C, Ory V, Candelier M, Baia M, Lang P, Pawlak A and Sahali D: Occurrence of minimal change nephrotic syndrome in classical Hodgkin lymphoma is closely related to the induction of c-mip in Hodgkin-Reed Sternberg cells and podocytes. Blood 115: 3756-3762, 2010.

14. LiuY,SuL,LinQ,HanY,YouPandFanQ:InductionofC-MipbyIL-17 plays an important role in Adriamycin-induced Podocyte damage. Cell Physiol Biochem 36: 1274-1290, 2015.

15. Ory V, Fan Q, Hamdaoui N, Zhang SY, Desvaux D, Audard V, Candelier M, Noel LH, Lang P, Guellaën G, et al: c-mip down-regulates NF- $\kappa \mathrm{B}$ activity and promotes apoptosis in podocytes. Am J Pathol 180: 2284-2292, 2012

16. Scerri TS, Morris AP, Buckingham LL, Newbury DF, Miller LL, Monaco AP, Bishop DV and Paracchini S: DCDC2, KIAA0319 and CMIP are associated with reading-related traits. Biol Psychiatry 70: 237-245, 2011.

17. Fletcher CDM, Unni KK and Meretens F (eds): Pathology and Genetics of Tumours of Soft Tissue and Bone. In: World Health Organization Classification of Tumors. IARC Press, Lyon, 2002. 
18. Ding K, Wu Z, Wang N, Wang X, Wang Y, Qian P, Meng G and Tan S: MiR-26a performs converse roles in proliferation and metastasis of different gastric cancer cells via regulating of PTEN expression. Pathol Res Pract 213: 467-475, 2017.

19. Capes-Davis A, Theodosopoulos G, Atkin I, Drexler HG Kohara A, MacLeod RA, Masters JR, Nakamura Y, Reid YA, Reddel RR, et al: Check your cultures! A list of cross-contaminated or misidentified cell lines. Int J Cancer 127: 1-8, 2010.

20. Jiang T, Zhao B, Li X and Wan J: ARPP-19 promotes proliferation and metastasis of human glioma. Neuroreport 27: 960-966, 2016.

21. Ding ZY, Jin GN, Wang W, Chen WX, Wu YH, Ai X, Chen L, Zhang WG, Liang HF, Laurence A, et al: Reduced expression of transcriptional intermediary factor 1 gamma promotes metastasis and indicates poor prognosis of hepatocellular carcinoma. Hepatology 60: 1620-1636, 2014

22. Livak KJ and Schmittgen TD: Analysis of relative gene expression data using real-time quantitative PCR and the 2(-Delta Delta C(T)) method. Methods 25: 402-408, 2001

23. Hurt EM, Wiestner A, Rosenwald A, Shaffer AL, Campo E, Grogan T, Bergsagel PL, Kuehl WM and Staudt LM: Overexpression of c-maf is a frequent oncogenic event in multiple myeloma that promotes proliferation and pathological interactions with bone marrow stroma. Cancer Cell 5: 191-199, 2004

24. Nip H, Dar AA, Saini S, Colden M, Varahram S, Chowdhary H, Yamamura S, Mitsui Y, Tanaka Y, Kato T, et al: Oncogenic microRNA-4534 regulates PTEN pathway in prostate cancer. Oncotarget 7: 68371-68384, 2016.

25. Bartel DP: MicroRNAs: Genomics, biogenesis, mechanism, and function. Cell 116: 281-297, 2004.

26. Yoo HI, Kim BK and Yoon SK: MicroRNA-330-5p negatively regulates ITGA5 expression in human colorectal cancer. Oncol Rep 36: 3023-3029, 2016.

27. Zhang Y, Peng Z, Zhao Y and Chen L: microRNA-25 inhibits cell apoptosis of human gastric adenocarcinoma cell line AGS via regulating CCNE1 and MYC. Med Sci Monit 22: 1415-1420, 2016.

28. Xin R, Bai F, Feng Y, Jiu M, Liu X, Bai F, Nie Y and Fan D: MicroRNA-214 promotes peritoneal metastasis through regulating PTEN negatively in gastric cancer. Clin Res Hepatol Gastroenterol 40: 748-754, 2016.

29. Li W, Zhang J, Chen T, Yin P, Yang J and Cao Y: miR-132 upregulation promotes gastric cancer cell growth through suppression of FoxO1 translation. Tumour Biol: Aug 23, 2015 (Epub ahead of print).

30. Wang Y, Zeng J, Pan J, Geng X, Li L, Wu J, Song P, Wang Y, Liu J and Wang L: MiR-320a inhibits gastric carcinoma by targeting activity in the FoxM1-P27KIP1 axis. Oncotarget 7 : 29275-29286, 2016.

31. Xu C, Li M, Zhang L, Bi Y, Wang P, Li J and Jiang X: MicroRNA-205 suppresses the invasion and epithelial-mesenchymal transition of human gastric cancer cells. Mol Med Rep 13: 4767-4773, 2016.

32. Tsai MM, Huang HW, Wang CS, Lee KF, Tsai CY, Lu PH, Chi HC, Lin YH, Kuo LM and Lin KH: MicroRNA-26b inhibits tumor metastasis by targeting the KPNA2/c-jun pathway in human gastric cancer. Oncotarget 7: 39511-39526, 2016.
33. Liu P, Ye F, Xie X, Li X, Tang H, Li S, Huang X, Song C, Wei W and Xie X: mir-101-3p is a key regulator of tumor metabolism in triple negative breast cancer targeting AMPK. Oncotarget 7: 35188-35198, 2016

34. Liu XY, Liu ZJ, He H, Zhang C and Wang YL: MicroRNA-101-3p suppresses cell proliferation, invasion and enhances chemotherapeutic sensitivity in salivary gland adenoid cystic carcinoma by targeting Pim-1. Am J Cancer Res 5: 3015-3029, 2015.

35. Sheng Y, Li J, Zou C, Wang S, Cao Y, Zhang J, Huang A and Tang H: Downregulation of miR-101-3p by hepatitis B virus promotes proliferation and migration of hepatocellular carcinoma cells by targeting Rab5a. Arch Virol 159: 2397-2410, 2014.

36. Cui W, Wu R, Cao H, Gao J, Wang X and Ren Q: P53 gene mutation and expression of MDM2, P53, P16 protein and their relationship in human glioma. J Huazhong Univ Sci Technolog Med Sci 25: 622-624, 635, 2005

37. Danilovskyi SV, Minchenko DO, Moliavko OS, Kovalevska OV, Karbovskyi LL and Minchenko OH: ERN1 knockdown modifies the hypoxic regulation of TP53, MDM2, USP7 and PERP gene expressions in U87 glioma cells. Ukr Biochem J 86: 90-102, 2014.

38. Shen J, Niu W, Zhou M, Zhang H, Ma J, Wang L and Zhang H: MicroRNA-410 suppresses migration and invasion by targeting MDM2 in gastric cancer. PLoS One 9: e104510, 2014

39. Wang BY, Cao J, Chen JW and Liu QY: Triptolide induces apoptosis of gastric cancer cells via inhibiting the overexpression of MDM2. Med Oncol 31: 270, 2014.

40. Saji S, Nakashima S, Hayashi S, Toi M, Saji S and Nozawa Y: Overexpression of MDM2 in MCF-7 promotes both growth advantage and p53 accumulation in response to estradiol. Jpn J Cancer Res 90: 210-218, 1999.

41. Hai J, Sakashita S, Allo G, Ludkovski O, Ng C, Shepherd FA and Tsao MS: Inhibiting MDM2-p53 interaction suppresses tumor growth in patient-derived non-small cell lung cancer Xenograft models. J Thorac Oncol 10: 1172-1180, 2015.

42. Wei L, Li Y and Suo Z: TSPAN8 promotes gastric cancer growth and metastasis via ERK MAPK pathway. Int J Clin Exp Med 8: 8599-8607, 2015.

43. Yang M, Gu YY, Peng H, Zhao M, Wang J, Huang SK, Yuan XH, Li J, Sang JL, Luo Q and Huang C: NAIF1 inhibits gastric cancer cells migration and invasion via the MAPK pathways. J Cancer Res Clin Oncol 141: 1037-1047, 2015.

44. Zhao M, Howard EW, Parris AB, Guo Z, Zhao Q and Yang X: Alcohol promotes migration and invasion of triple-negative breast cancer cells through activation of p38 MAPK and JNK. Mol Carcinog 56: 849-862, 2017.

45. Kim GT, Lee SH and Kim YM: Torilis japonica extract-generated intracellular ROS induces apoptosis by reducing the mitochondrial membrane potential via regulation of the AMPK-p38 MAPK signaling pathway in HCT116 colon cancer. Int J Oncol 49: 1088-1098, 2016.

46. Peng W and Fan H: Long noncoding RNA CCHE1 indicates a poor prognosis of hepatocellular carcinoma and promotes carcinogenesis via activation of the ERK/MAPK pathway. Biomed Pharmacother 83: 450-455, 2016.

47. Yokozaki H: Molecular characteristics of eight gastric cancer cell lines established in Japan. Pathol Int 50: 767-777, 2000. 